\title{
„DAS UNTERHALTSAME EXOTIKUM“: DIE ROLLE EINIGER UNGARISCHEN VOLKSGRUPPEN IM FREMDENVERKEHR DER ZWISCHENKRIEGSJAHRE
}

\author{
Márta FÜGEDI \\ Lehrstuhl für Kulturgeschichte und Museologie \\ H-3515 Miskolc-Egyetemváros, Ungarn
}

Die modernen Organisationsrahmen und das Institutionsnetz des ungarischen Fremdenverkehrs wurden in den 1920-er Jahren ausgestaltet. Im Jahre 1929 wurden das Fremdenverkehrsbüro und Fremdenverkehrsrat gegründet. ${ }^{1}$ Die Expansion des Tourismus zielte bald auch auf die Volkskultur, unter den Anziehungskräften des Fremdenverkehrs spielten nämlich das ungarische Dorf und die Volkskunst immer größere Rolle. Obwohl sich die Volkskultur in Ungarn nach dem ersten Weltkrieg und dem Frieden in Trianon in einer starken gesellschaftlichen, wirtschaftlichen und moralischen Krise und Verwandlung befand, gab es doch fertige und billige Möglichkeiten, die der Fremdenverkehr wohl nutzen konnte.

Man hat damit schon früher begonnen, die für ihre bunte spektakuläre Volkskunst berühmten Gegende zu entdecken, sich ihr Hausgewerbe anzunehmen und kommerziell zu nutzen, so sind die spektakulären und sinnlichen Gegenstände und die Festbräuche im Vornherein zum Zielpunkt für den organisierten Fremdenverkehr ${ }^{2}$ (Abb. 1) geworden.

Am Beginn der 1930-er Jahre hat man angefangen, das Fremdenverkehrgesicht dieser frequentierten Gegende bewußt zu formen, sie in den organisierten Touristenprogrammen einzuschalten, sowie das dazu nötige Institutionsnetz auszubauen. Der Staatssekretär, Géza Tormay, der auch der Vorsitzende des National-büros für Fremdenverkehr war, schrieb in seinem Artikel „Volkskunst und Fremdenverkehr“ im Jahre 1937 folgende 3 : ,....nicht nur in Budapest und in den größeren ländlichen Städten, sondern auch in den Gegenden, wo Folklore wichtig und lebendig ist, müssen Gästehäuser mit Herbergen gegründet werden, wo die charakteristischen Volkskunstschätze der Umgebung gesammelt werden, und so die ausländischen Gäste nach der Besichtigung ein richtiges Bild bekommen können...".

In den Gegenden, die für ihre spektakuläre Volkskunst und Hausgewerbe berühmt waren, wurden Gasthäuser ausgestattet, die als Rahmen zum Empfang der Gäste und zur Vorstellung der lokalen Werte und Sehenswürdigkeiten dienten. Diese Gasthäuser erfüllen also eine komplexe Funktion:

1 FEJÖS 1984: 40.

2 FEJŐs 1991: 143-158; K. CSILLÉRY 1983: 353-382; HOFER 1989: 59-84.

3 TORMAY 1937: 86-87. 


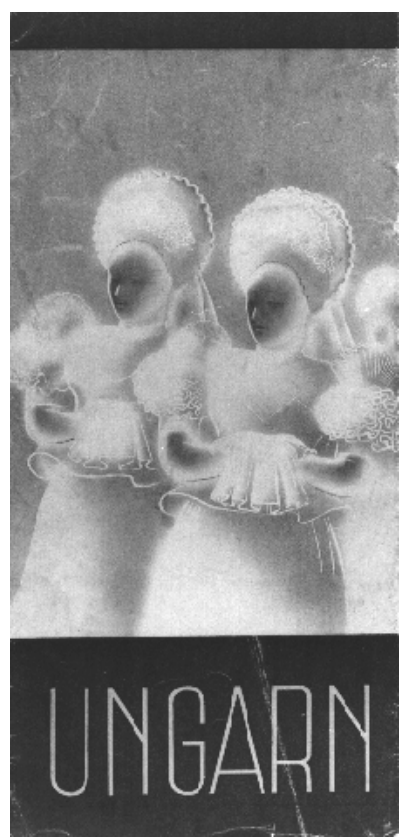

Abb. 1. „Ungarn“: Deutschsprachiges Programmheft aus den 1930-er Jahren. Titelblatt mit der Volkstrachts-Figur der sog. Marienmädchen von Matyóland

1. sie müssen ein volkisches und „gut ungarisches“ Milieu eingeben, deshalb zeigt die Einrichtung und Umgebung den Eindruck eines Bauernhauses der gegebenen Volksgruppe - mit bunt gemalten Möbelstücken, Stickereien, Töpferwaren und mit dem Bauernofen, sie alle erwecken die Illusion der Bauernstube.

2. Gasthäuser erfüllen gleichzeitig eine Gastgeberfunktion, sie müssen dazu auch geneigt sein, eine größere Menge von Gästen zu empfangen und zu beherbergen.

3. Die Gasthäuser funktionieren zugleich auch als Museen und Geschäfte, denn das Angebot an Souvenieren und die konzentrierte, verkürzte Vorstellung der lokalen Volkstradition (Volksbräuche und Tänze) ist ein unerläßlicher Teil der Dienstleistung Fremdenverkehr.

Das erste Gasthaus für Fremdenverkehr wurde 1933 in Mezókövesd, ,in der Hauptstadt der Matyós“ eröffnet. Es wird von der zeitgenössischen Presse zuerst als „Ungarische Tscharda“, dann als „Fremdenhaus“ oder „Gasthaus“ erwähnt, bald auch allgemein und einfach nur „Matyóhaus“ genannt. ${ }^{4}$

„Die unvergänglich schätzlichen Stücke der unverfälscht ungarischen Volkskunst von Sárköz“ in Bezirk Tolna (Südwestungarn), besonders die Produkte der Weberkunst erhielten schon 1926 an der Weihnachtsausstellung des Hausgewerb-

${ }^{4}$ FÜGEDI 1997: 127-140. 


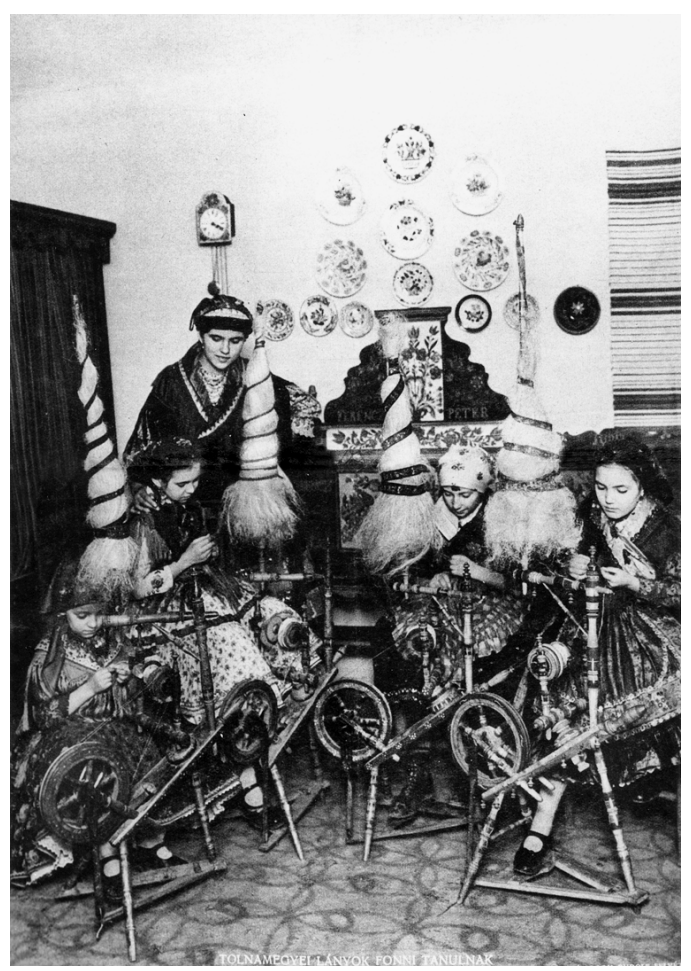

$A b b$. 2. Kleine Mädchen spinnen in dem Gästehaus von Sárköz. Illustrierter Bericht in der Zeitschrift Pesti Napló. Photo: Rudolf Balogh

lichen Nationalvereins in der Hauptstadt einen Publikumserfolg. In der Siedlung Decs in Sárköz wurden die interessierten Fremden von der seit 1929 tätigen Hausgewerbegenossenschaft empfangen, aber auch in der Bezirkstadt Szekszárd wurden ständig Volkskunstausstellungen und Märkte für Fremdenverkehrszwecke veranstaltet (Abb. 2). ${ }^{5}$

Weitere stark gewerbte „Volkskunstzielpunkte“ waren vor allem Kalocsa, Boldog, Tihany und Kiskunhalas.

In Kalocsa wurde im Jahre 1936 „das Volkskunsthaus“ eingerichtet, wo neben den bunt bemalten Möbelstücken auch die Wandmalerei (ung. „pingálás“), d.h. der Anstrich hervorgehoben wurde. ${ }^{6}$ Das mit der verzierten Bettwäsche gedeckte Bett, die Möbelstücke mit den bunten Blumenmustern, die gleicherweise bunt verzierte Volkstracht und natürlich der wuchernde Anstrich auf den Wänden zusammen brachten dem Betrachter - laut der zeitgenössischen Prospekte - „die Farbenpracht der ungarischen Blumenwiese und der Frühjahrsfelder“ in Erinnerung (Abb. 3).

${ }^{5}$ FLÓRIÁN 1990: 210-223; SZILÁGYI 1980:15-21.

${ }^{6}$ K. CSILLÉRY 1983: 353-382. 


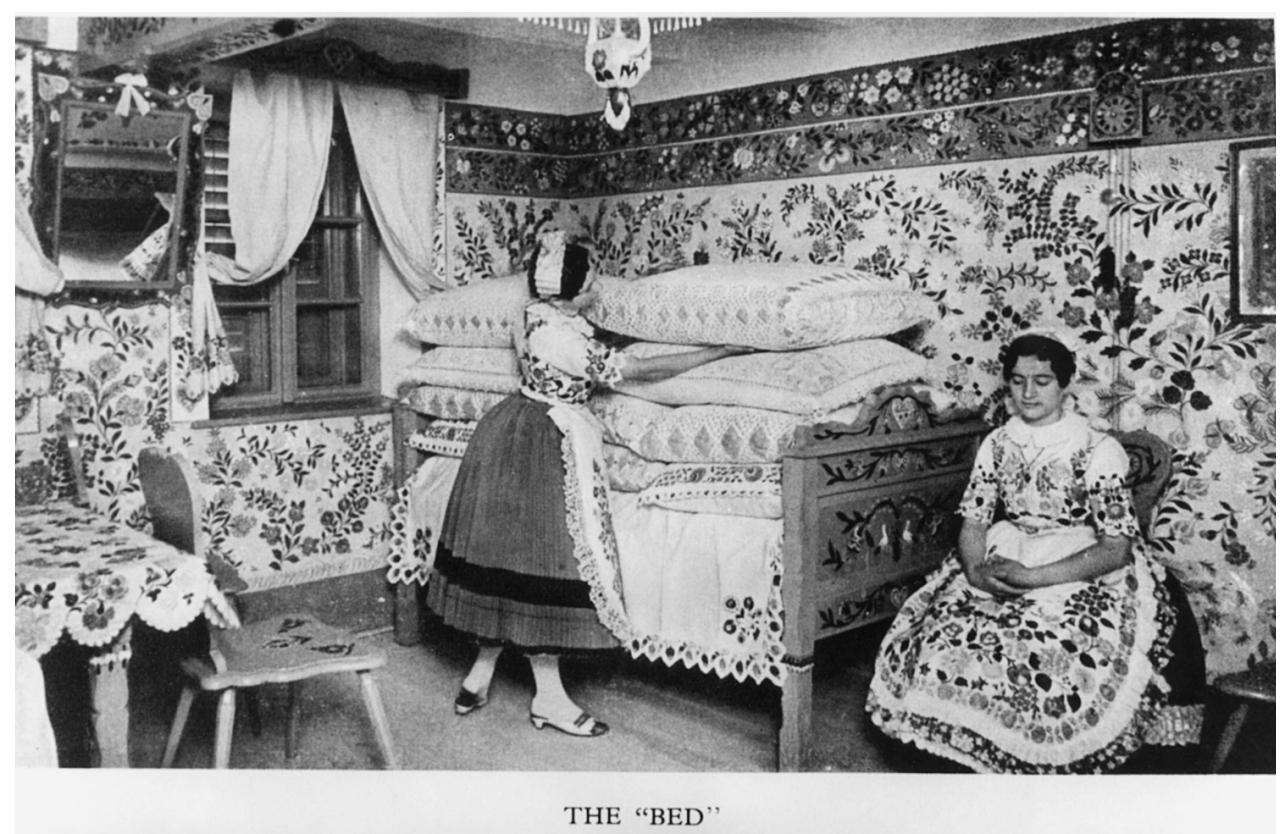

Abb. 3. „Das Bett“. Ein Bild aus dem Gästehaus von Kalocsa. Photo: Magyar Filmiroda (Ungarisches Filmbüro)

In Boldog, in der Nähe der Hauptstadt, wurde das Gasthaus der Kirche gegenüber 1936 eröffnet. ${ }^{7}$ Die Besichtigung des Dorfes, das auch von der spektakulären Volkstracht berühmt ist, wurde vor allem als Sonntagsprogramm empfohlen. Eine beachtenswerte Programmverbindung für Touristen war die Besichtigung von Boldog und nachdem die des Barock-Schlosses von Gödöllő in der Nähe.

Der Mitarbeiter des Ethnographischen Museums in Budapest, dr. Sándor Ébner, war einer von denen, die die Volkskunst von Boldog am begeistertsten propagierten. Im September 1930 schrieb er dem Notar von Boldog die folgenden: „Das Volk von Boldog verdient es wohl, daß sich die Welt von ihm Kenntnis verschafft, und meinerseits werde ich das Fremdenverkehrsbüro und das Geschäft des Ungarischen Hausgewerbes darauf aufmerksam machen, daß sie die Fremden nicht nur nach Mezökövesd schicken, sondern auch das Volk von Boldog ihnen vorstellen sollen. “8 (Abb. 4).

In Kiskunhalas (auf dem ungarischen Tiefland) wurde das Gasthaus mit Fremdenverkehrszwecken „Spitzenhaus“ genannt, wo die Gäste die Nagelspitze - als eine Rivale der Brüsseler Spitze ausgerufen - kennenlernen konnten. ${ }^{9}$ Die Spitze von

7 ÚJVÁRI 1981: 21-36.

8 ÚJVÁRI 1981: 28.

${ }^{9}$ Új Idők (Neue Zeiten), 19. Febr. 1939. 


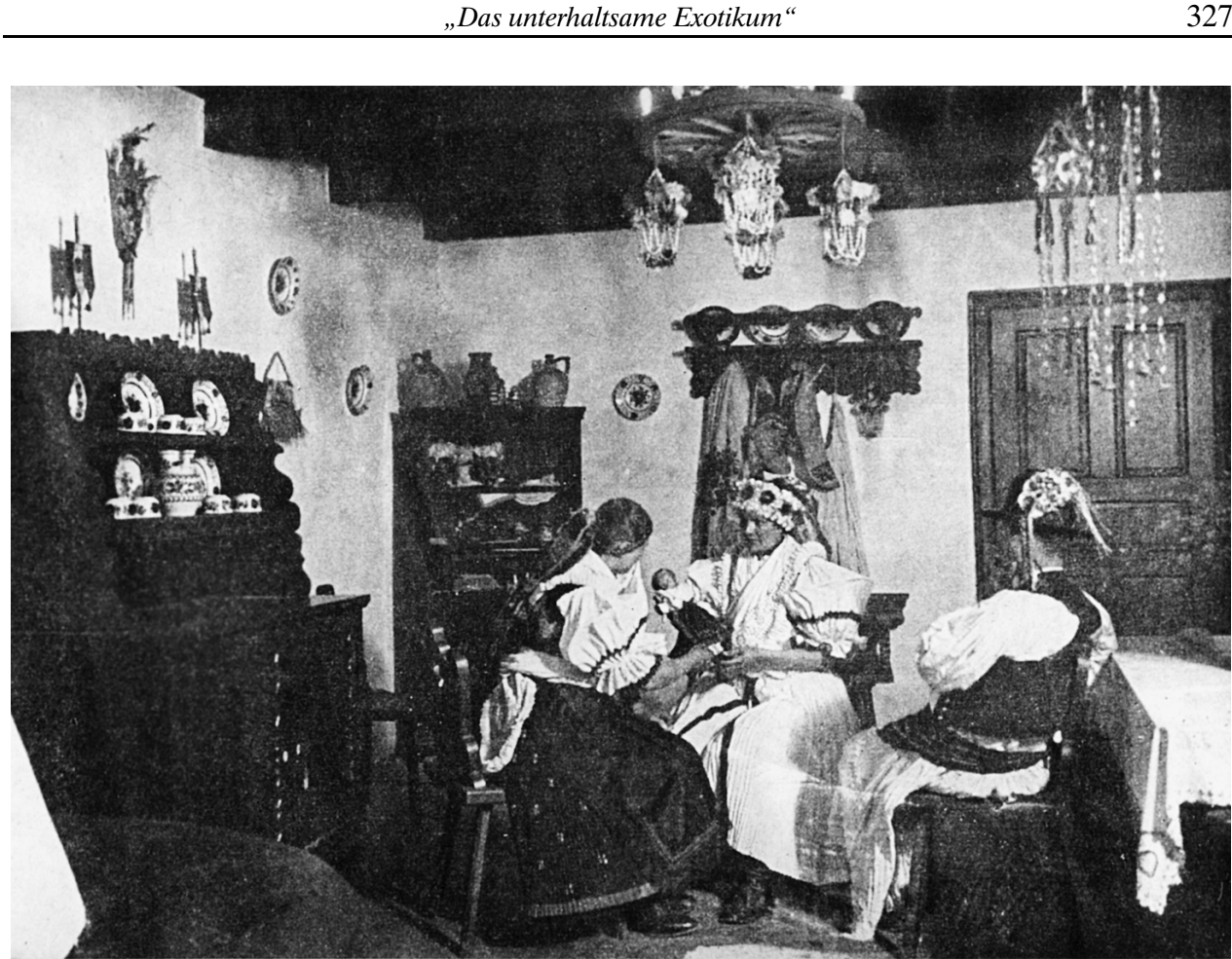

$A b b$. 4. Gästehaus in Boldog. Photo: Ernő Vadas. Illustrierter Bericht in der Zeitschrift Új Idôk (Neue Zeiten), Jan. 8. 1939.

Halas schloß sich in den 1920-er Jahren ans Angebot „der alten Werte der ungarischen Volkskunst", trotzdem, daß sie erst an der Jahrhundertwende im Zeichen der ungarischen Sezession ihre Laufbahn begann, und eigentlich auf Grund der Entwürfe eines Kunstlehrers entstanden ist (Abb. 5).

Auf die obenerwähnten „repräsentativen Gegende“ wurden die Interessierten, die Touristengruppen mit Sonderbussen transportiert, ab 1932 gab es eine begünstigte Fahrtmöglichkeit, das Netz der sog. Groschenzüge, mit denen die Gäste zu einem ermäßigten Preis zum Ziel gelangen konnten (Abb. 6).

Die Volkskunstausstellungen in den Gasthäusern, die Verkaufsmöglichkeiten für Touristen gestalteten selbst die lokalen Traditionen und die Meinung der Bevölkerung über seine eigene Kultur. Die Volkstradition wurde eigentlich ein wichtiger Teil der Dienstleistungen im Fremdenverkehr. Die Arbeit der Organisation und die Leitung des Gasthauses war in der Hand der lokalen Intelligenz. ${ }^{10}$

Die lebendige Volkstradition aber zeigte sich zu dieser Zeit in den verwandelnden ungarischen Dörfern meistens nur an den Festtagen. Die Festtracht, die man in der Kirche trug, die bunte Menge von Dorfsleuten auf der Straße am Sonntag oder

${ }^{10}$ FÜGEDI 1999. 


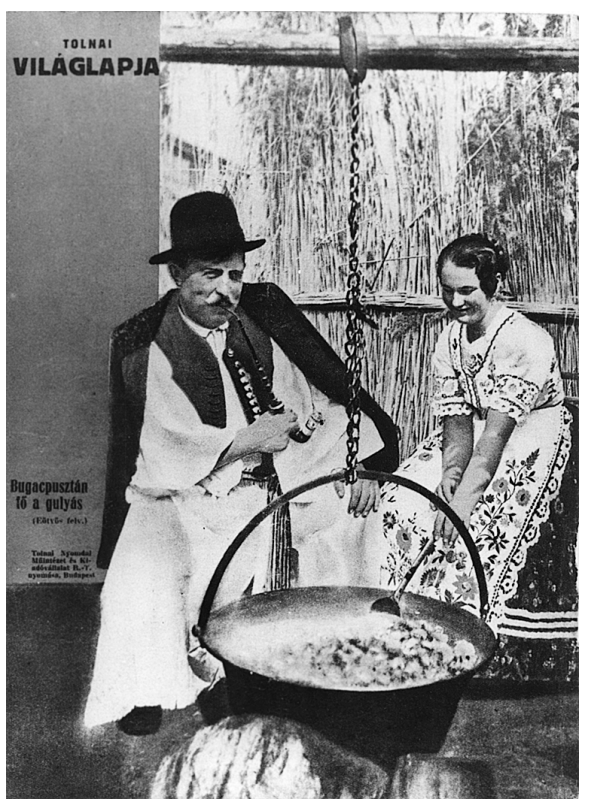

$A b b$. 5. Ungarischer Gulasch in Bugac-Pussta. Titelblatt in der Zeitschrift Tolnai Világlapja (Tolnais Weltblatt). Okt. 14. 1936.

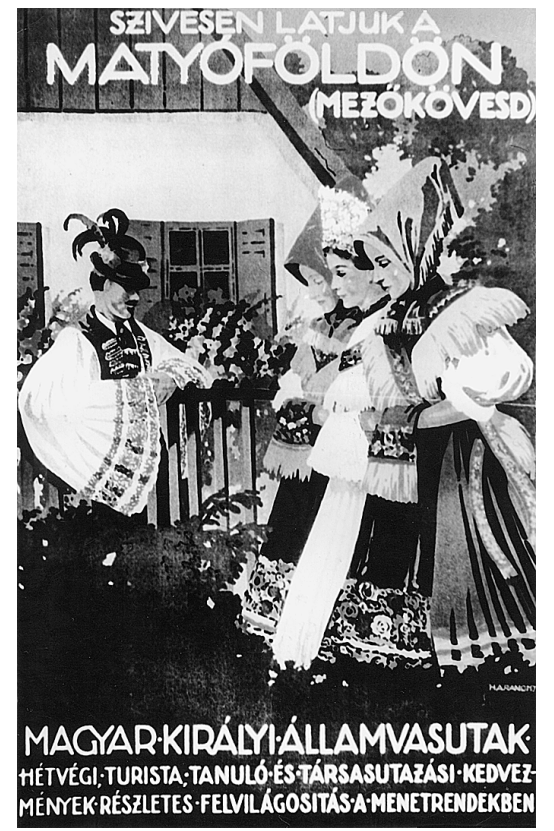

$A b b$. 6. Willkommen auf dem Matyóland! Ein koloriertes Plakat der Ungarischen Staatsbahn (Magyar Királyi Államvasutak) 
die imposante Menschenmenge der Prozessionen und der Kirmessen bat intensiv und spektakulär das gesuchte „Volkserlebnis“. Es ist also kein Zufall, daß das Programmheft des Fremdenverkehrbüros IBUSZ in den 1930-er Jahren mit dem Titel „Der ungarische Sonntag“ erschien, und das Kennenlernen der „urwüchsigen ungarischen Stimmung“ an den Festtagen in den ungarischen Dörfern empfiehlt: „Sonntag morgen, als sie von der Messe kommen, kann sich der fotographierende Fremde an einer prunkvollen Orgie der Farben ergötzen, weswegen lohnt es sich, aufs Lande zu fahren“ - wird in einer Werbeschrift „Borsoder Panorama 1937“" geschrieben. ${ }^{11}$

In den Charakterzügen des ungarischen Fremdenverkehrs zu dieser Zeit spielten die Volkstraditionen und das ländliche Ungarn auch aus einem anderen Aspekt eine wichtige Rolle. Das wirkungsvolle Motiv „Ungarn zwischen Westen und Osten"12 ist ein ständiger Slogan und Gedankenkreis zur Zeit der Zwischenkriegsjahren in den Fremdenverkehrsprospekten. Zu dieser Zweigesichtlichkeit können alle Werte gezählt werden, die man im Fremdenverkehr zu betonen wünschte; zB. das Ungartum ist einerseits Europas Schutzschild gegen die östlichen Barbaren, andererseits ist es aber ein Aufbewahrer der alten östlichen Werte in Europa.

Das zur Weltstadt gewordene Budapest repräsentiert den Westen, das ländliche Ungarn, vor allem die Volksgruppen mit einer charakteristisch lokalen Kultur und die einzelnen Naturwerte repräsentieren die östliche Erbe, natürlicherweise in einem friedlichen Zusammenleben. In einer deutschsprachigen Werbeschrift aus dem Jahre 1934 wird diese Doppelheit gerade als Motto benutzt: „östlicher Prunk in westlicher Umgebung.“

Ein glückliches Zusammentreffen der als uralt erkannten Volkstraditionen und der modernen Werte von Budapest, als eine Weltstadt und ein Fremdenverkehrszentrum war der „Perlenstrauss“, der sich in den 1930-er Jahren zu einer Landbewegung ausbreitete. ${ }^{13}$ Die dörflichen Volksgruppen, die die Volkstraditionen, den Volkstanz, die Volkslieder und die Volksbräuche erneuerten, wurden von der die Nationaltraditionen betonenden Intelligenz und der Staatsregierung unterstützt, und die Bewegung erhob sich als eine Fremdenverkehrsattraktion. Das große Ereignis des Perlenstrausses war jedes Jahr der große Aufzug am 20-sten August in Budapest, wo der Ruf und die Anziehungskraft des zum Nationalfeiertag gewordenen St. Stephan Tages von den Bauergruppen des Perlenstrausses durch ihre spektakuläre und doch billige Produktionen erhöht wurde. Das Tageblatt Pesti Hirlap (Pester Zeitung) berichtet 1935 entzückt über den Aufzug der Bauerngruppen in Volkstracht: „Der Blumengarten der ungarischen Felder strömt jetzt der Andrássy Straße entlang." ${ }^{14}$ Eine deutschsprachige Werbeschrift aus dem Jahre 1934 schildert folgendermaßen die Anziehungskraft der Perlenstrauss-Vorführungen, auch eine Volkstrachtenrevue genannt ${ }^{15}$ : (Abb. 7).

11 Alle Prospekte und Werbeschrifte in Besitz des Verfassers.

12 Hofer 1995: 7-22. und die weiteren Vorlesungen der Konferenz „Die Ungarn zwischen Osten und Westen".

13 PÁlfi 1970: 115-161; F. KÁROLYI o.J.

14 Pesti Hírlap (Pester Zeitung), 19. Aug. 1935.

15 St. Stefans-Fest, Budapest, Ungarn. 1934. 


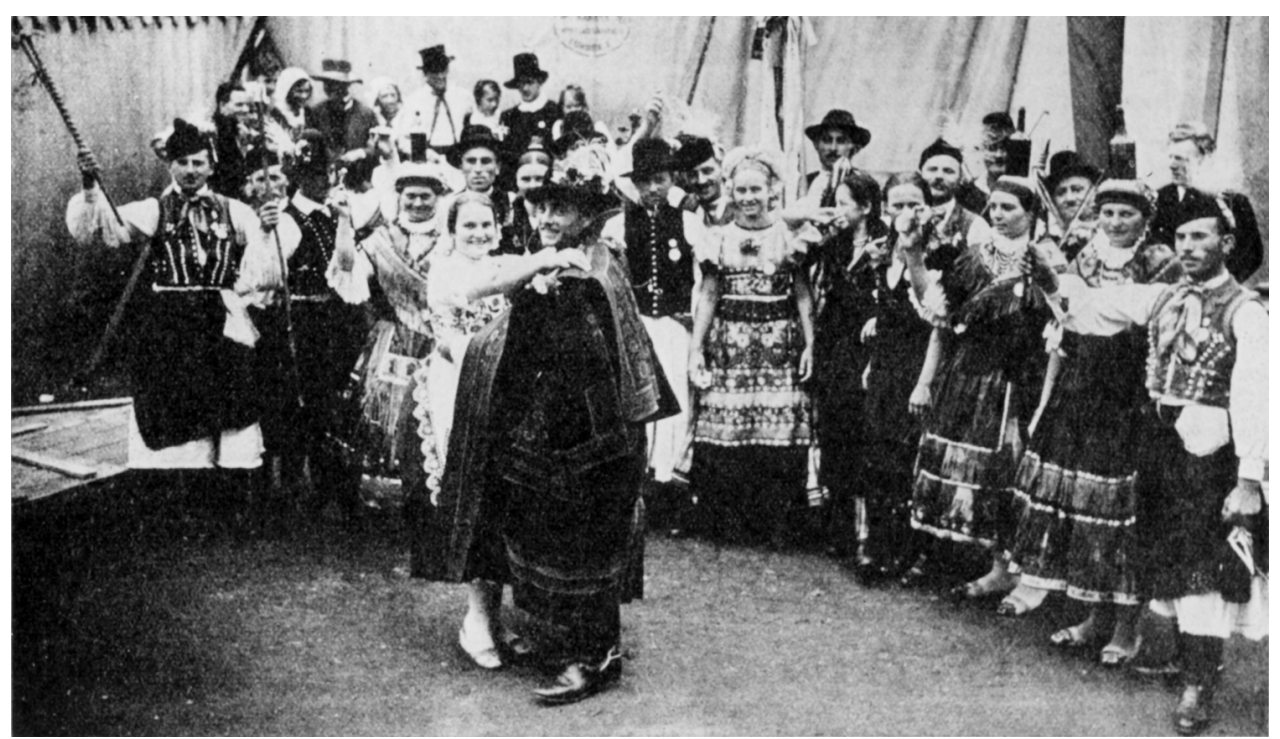

$A b b$. 7. Eine Perlenstrauss-Gruppe in London: ungarische Tänze in Regent-Park. Új Idők (Neue Zeiten), Aug. 4. 1935 .

„Der Perlenstrauss ist ein Festspiel, zu dem sich alljährlich aus allen Gegenden Ungarns die Blüte des ungarischen Volkes zusammenschart, um von seinem östlichen Urmagyarentum Zeugenschaft abzulegen. Der Perlenstrauss ist Festesfreude: das ungarische Volk stellt sich selbst dar und offenbart vor dem begeisterten vornehmen inländischen und ausländischen Publikum seine eigene Seele.“

Es geschah also in Ungarn in den 1930-er Jahren, daß bestimmte, für repräsentativ gehaltene Elemente der ungarischen Volkskultur und die ausgewählten Volksgruppen in das Fremdenverkehrsangebot bewußt eingefügt wurden. Das charakteristische Beispiel, das gleichzeitig auch allgemeingültige Lehren aufweist, ist der Prozeß, wie die Volksgruppe Matyó in Nordostungarn und „die Matyó-Hauptstadt“ Mezőkövesd zum Fremdenverkehrszentrum wurde. ${ }^{16}$

Das Matyótum konnte in die Profilausgestaltung und -ausbildung des organisierten Fremdenverkehrs aus mehreren Aspekten günstig und erfolgreich eingereiht werden. Das Matyóland hat nämlich eine günstige geographische Lage, es liegt einerseits nicht weit von der Hauptstadt, und hat andererseits günstige Verkehrsbedingungen, so wurde Mezókövesd zu einem beliebten Zielpunkt eintägiger Ausflüge. Neben der Pussta von Hortobágy und der Weingegend von Erlau wurde es ein Teil des Programms, den „Fremden ein vielseitiges Bild von Ungarntum“ anzubieten: Naturschönheit, Folklorerlebnisse und spezielle ungarische Gastfreundlichkeit mit dem weltberühmten Erlauer Stierblut konnten so die vielseitige Werte des ungarischen Fremdenverkehrs nebeneinander repräsentieren.

16 FÜGEDI 1997: 127-140. 
Das Fremdenverkehrsbüro IBUSZ schickte jeden Sonntag Gemeinschaftsausflüge für individuelle Gäste von Budapest (Abfahrtsort: Vigadó) nach Matyóland. Parallel mit der Erhöhung der Ansprüche hielt der sog. Groschenexpreß (BudapestMiskolc) der Ungarischen Staatsbahn auch in Mezőkövesd. Der Fremdenverkehr wurde in Mezókövesd ab 1933 institutionalisiert und organisiert, als es der Staatsbahn und dem Fremdenverkehrsbüro gelungen ist, die lokale Struktur auszubauen. $\mathrm{Zu}$ dieser Zeit wurde das Gasthaus, die charakteristische Fremdenverkehrsanlage von Mezőkövesd eröffnet. Das Gasthaus, lieber allgemein Matyóhaus genannt, wurde als ein charakteristisches Kennzeichen anerkannt, wo alles Interessante betrachtet werden konnte. „Die geschnitzten und bemalten Bauernmöbel und Leuchter, die künstlerischen Töpferwaren an der Wand, die Bediener in Matyó-Tracht erschließen uns den ungarischen Geschmack dieser Siedlung" - heißt es in dem Buch für Reisenden. ${ }^{17}$ (Abb. 8-9).

Die Einrichtung des Matyóhauses wurde von einem dortigen Tischlermeister, József Berecz, aber auf Grund der Entwürfe einer Kunstgewerblerin, Gitta Mallász gefertigt. Die Möbel - eine lange Armbank an der Wand, ein Spiegel, eine Wiege, Blumenhälter, kleine und größere Tische und Amtsessel, Bauerstellage, alle mit bunten Blumen bemalt - ließen die Atmosphäre der Matyó-Bauernmöbel und des Penkels ahnen, daneben diente das Gasthaus wegen der Räumlichkeit und der innerlichen Höhe der Säle sowie der zusammengesetzten Funktion der Bewirtung eher als eine Art Kulisse. Die Wände wurden ebenso wie in einer Bauernstube mit prächtigen Schmucktellern, Heiligenbildern und Familienfotos verziert, neben ihnen waren aber auch die Hetzpeitsche, prächtige gestickte Bauernmäntel, Lebkuchenherzen, Bettwäschestickereien an die Wand gehängt, als Zeichen des ungarischen Geschmacks, als eine Art Dekoration.

Die Hauswirtin des Gasthauses war die Frau eines Obersten (Frau Pöcher geb. Ilona Ibrányi-Vay), die mehrere Fremdsprachen beherrschte und ihre Aufgabe mit großer Hingabe versah. Über ihre Tätigkeit schrieb man in der Mezókövesder Zeitung belobend: „Frau Pöcher tut alles, um die Matyós auf den richtigen alten Weg zurückzuführen. Von Zeit zu Zeit ruft sie Matyó-Mädchen und Jungen zu sich, sie borgt ihnen die alten Volkstrachtstücke im Matyóhaus, und die bewirtete Jugend tanzt die alten Tänze, unterhält sich auf Matyó-Lieder. Oft gibt es ein großes Publikum bei diesen Matyó-Bewirtungen, und viele Fremde können mit unvergeßlichen Erlebnissen wegfahren."18

In der Gestaltung des „Matyó-Image“ bekam der Bahnhof von Mezőkövesd eine wichtige Rolle, der wegen der ersten Eindrücke der mit dem Zug ankommenden Gäste ein wichtiger Punkt des gewußt ausgestalteten Fremdenverkehrsgesichts wurde. Diese Aspekte vor dem Augen haltend kleidete die Ungarische Staatsbahn zusammen mit dem Gasthaus auch den Bahnhof „,in Matyó-Kleider“. Der Wartesaal wurde mit rot bemalten Matyó-Möbelstücke (Tische, Bänke, Kleiderhaken, Holzleuchter und Bilderrahmen) eingerichtet, noch dazu wurde auch ein Bauernofen in

\footnotetext{
${ }^{17}$ Az utas könyve. (Buch der Reisenden.) Budapest, 1935. 530.

${ }^{18}$ Felsómagyarország (Oberungarn), 19. Nov. 1933.
} 

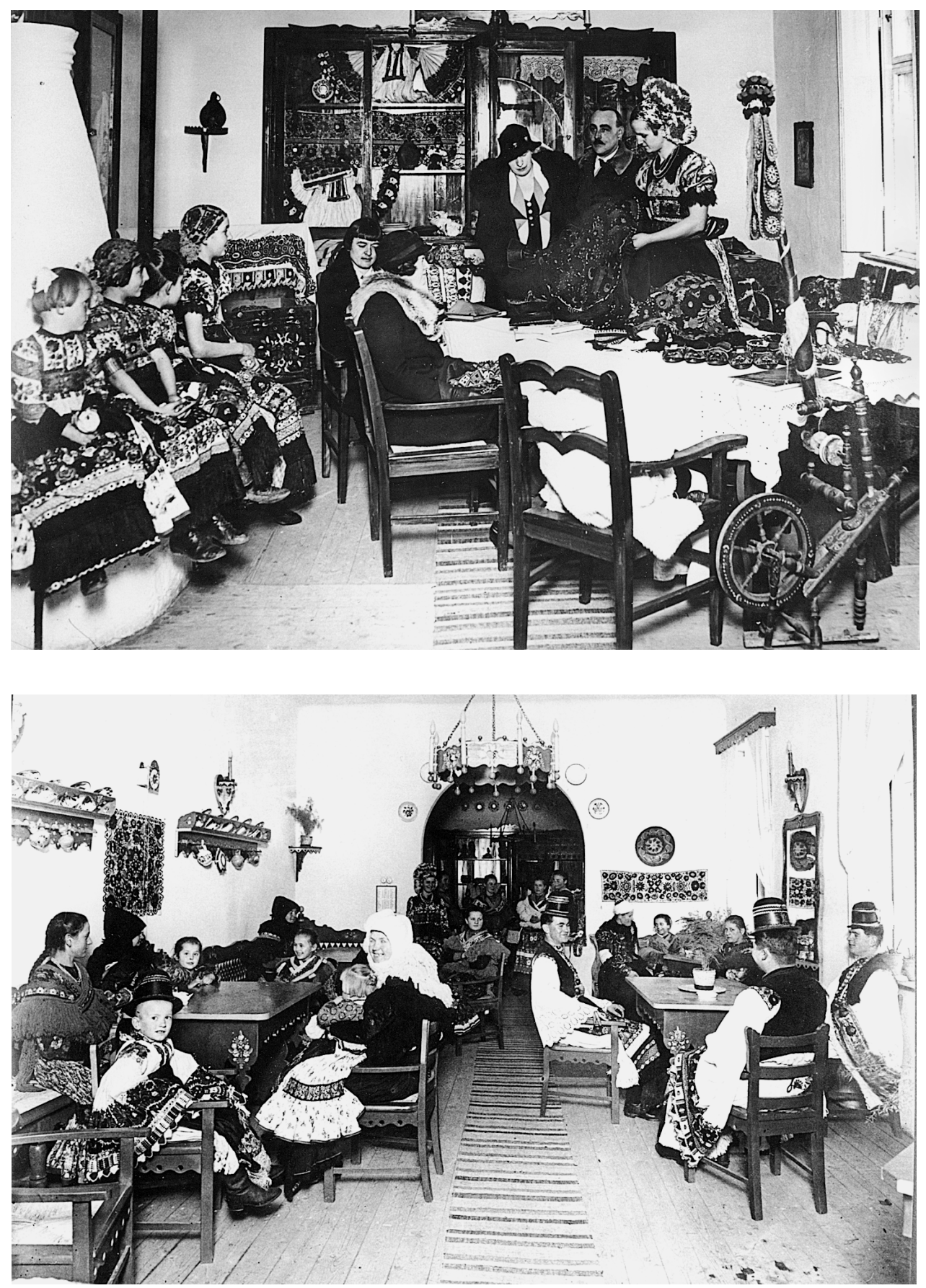

Abb. 8-9. Gäste und Gastgeber im „Matyóhaus“, in dem Gästehaus in Mezőkövesd. Photo: Magyar Filmiroda (Ungarisches Filmbüro) 
den Saal eingebaut. „Auf dem „Volkskunstbahnhof“ stehen noch auch prima mit Matyó-Blumen bemalten Gartenstühle im Sommer auf dem Bahnsteig unter roten Sonnenschirmen mit Matyó-Mustern" - schreibt man in einer Komitatssoziographie über das „Matyóland ${ }^{19 “}$.

Die Gastlichkeit und die Unterhaltung als Teil der Fremdenverkehrsleistungen im Matyóhaus verteilte bald stark die öffentliche Meinung der Siedlung: „...Die Staatsbahn richtete für ihre Gäste ein Gasthaus mit Matyó-Möbeln und Bauernofen ein, und kümmerte sich um ihre Unterhaltung. Hochgewachsene Matyó-Burschen in Gatya-Hosen und gesticktem Hemd, komische Hüte auf dem Kopf mit Kranzenkraut geschmückt sitzen auf dem Hof, es lautet Zigeunermusik. Die Mädchen in der buntesten Tracht warten auf den Tanz. Nach dem köstlichen Mittagessen der Gäste setzt die Musik ein, die Matyópaare drehen sich auf dem Beton herum. Ihre vielkräusigen Röcke öffnen sich wie Glocken und fliegen nach ihnen. Die weissen Unterröcke und die langen, unter Knie reichenden Unterhosen blinken auf. Die vielen Kräusen und Bänder schweben in der Luft und die weiten Gatya-Hosen fliessen hin und her. Es ist ja überaus exotisch für die fremden Menschengruppen, die erstaunend herumstehen. Die Burschen tanzen mit den ausländischen Damen. Die Mädchen nehmen die älteren Herren in den Tanz mit, drehen sie wohl auf Matyó Art" - beschreibt József Dala in einer Soziographie das Milieu, was dem „Matyó-Volkserlebnis“ suchenden Gast im Gasthaus angeboten wurde..$^{20}$

In einem Fremdenverkehrswerbungsheft „Wir warten auf Sie in Borsod!“ schreibt man folgende: „Die Veranda des Gasthauses ist sonntag nachmittags dem Molo einer Hafenstadt ähnlich. Fünf oder sechs Sprachen werden hier gesprochen, ein echtes heilloses Durcheinander ist zu hören, wenn die Autos und Autobusse ankommen. “21

Dagegen steht aber ein rücksichtsloses Bild über den Fremdenverkehr in Matyóland, das von Zoltán Szabó in seinem Werk „Glanzendes Elend“ geschildert ist: „Wie ein schrecklicher Gegensatz, daß gerade in der Mitte des armseligsten Matyó Dorfes eine Potemkin-Kneipe geschaffen wurde, wo die Pracht des falschen ungarischen Lebens zum Vergnügen der Fremden gezeigt wird... In der Fremdenverkehrstscharda werden Matyó-Mädchen und Matyó-Burschen zusammen mit Aprikosenschnaps serviert..." 22

Der Massentourismus, das Interesse der Besucher, sowie die lokalen Möglichkeiten formten bald das ständige Drehbuch des Fremdenverkehrsprogramms in Mezőkövesd aus. Die wichtigsten Punkte, die stereotypischen Motive des Angebotes, das Matyó Volksleben als charakteristisches, repräsentatives ungarisches Volksprogramm vorstellt, sind folgende:

1. „Die Besichtigung“ des Matyó Volkes - erlebnisvolle spontane Eindrücke über das Volksleben, vor allem sonntags, als die Bevölkerung die festliche Volks-

19 Kozma 1939: 108.

${ }^{20}$ DALA-ERDÉLYI 1941: 15.

${ }^{21}$ Miskolci Kirakat (Miskolcer Schaufenster). Miskolc, 1936.

22 SZABÓ 1938: 74. 
tracht anhat. Besonders sehenswürdig ist es zur Zeit des Kirchenganges und nachmittags, als die Mädchen Arm in Arm auf der Hauptstrasse spazierengehen. Die festliche Volkstracht als eine massenhafte Sehenswürdigkeit gibt wirklich einen echt bunten und entzückenden Anblick.

Prominente Persönlichkeiten und die sich fürs Volksleben tiefer interessierten Besucher blickten gern hinter die Kulissen, so wurden der Besuch einer ausgewählten Bauernfamilie in ihrem Wohnhaus und das direkte Kennenlernen des bäuerlichen Lebens- und Wohnungsumstände zu beliebtem Programme. ZB. die Großherzogin von Oldenburg besuchte das Bauernhaus von Márton Petô, wo sie auch den Stall betrachtete. ${ }^{23}$

2. Die stilisierte und dramatisierte Vorstellung der Volksbräuche wurde nach der erfolgreichen Matyó-Hochzeit von Millenniumsfest in Budapest ein ständiges Programm. Die Brautfestvorstellungen, die sich aus den Hochzeitsbräuchen herausbildeten, wurden seit dem Beginn des Jahrhunderts zu einer selbständigen Gattung. Die Hochzeitszenen im Gasthaus, auf der Strasse oder auf einer Freilichtbühne wurden bald von Bauerndarstellern, bald von Schulkindern oder von Laienschauspielern, mit weniger oder vielen Mitwirkenden dargestellt. Es war eigentlich eine Auswahl aus der Zeremonienreihe der traditionellen Hochzeit, in mehreren Variationen, von der kurzen Kammer-Version bis zur spektakulären Variation mit vielen Darstellern und Teilnehmern. In den Berichten der zeitgenössischen Zeitungen ist oft die folgende Abfassung zu lesen: „Lebensbilder aus dem Matyó Volksleben wurden vorgeführt“" oder: „Es wurde eine Volksunterhaltung den Gästen vorgezeigt.“

3. Bewirtung und Unterhaltung unter aktiver Zuziehung der Gäste. Die Verknüpfung des Fremdenverkehrs, der Besichtigung mit der Bewirtung und Konsumierung hat in Mezőkövesd seit der Eröffnung des Gasthauses einen gesicherten Hintergrund. Hier gab es eine Möglichkeit, 60-70 Gäste zu bewirten und ihnen mit Essen, Trinken und Tanz zusammengeknüpft eine dörfliche ungarische Unterhaltungsmöglichkeit zu bieten.

4. Die spektakuläre Volkstracht der Matyós zu erproben, die prächtige Kleidungsstücke anzuziehen und in Volkstracht Photos als Andenken machen lassen, das alles war früher das Erlebnis der privilegisierten, vornehmen Gäste, aber im Gasthaus hatten alle Gäste Möglichkeit, Volkstrachtstücke anzuprobieren. Die Großherzogin von Oldenburg, die Frau des Fürsten von Siam und der Erzherzog Joseph Franz mit seiner Frau, Herzogin Anna, erprobten alle die Matyó-Tracht, und ihre Trachtenphotos in den Zeitungen bedeuteten ein hervorragendes Propaganda für die Matyós (Abb. 10).

5. Die Vorstellung der Andenken und der Produkte der lokalen Volkskunst, vor allem die Stickereien und die Volkstracht waren natürlich ein unerlässlicher Programmpunkt für die Besucher. Die ethnographische Sammlung des Gymnasiums erfüllte deshalb die Rolle des Museums im Fremdenverkehr, wo die Besucher durch die Gegenstände und Dokumente die Vergangenheit und die ethnographische Cha- 


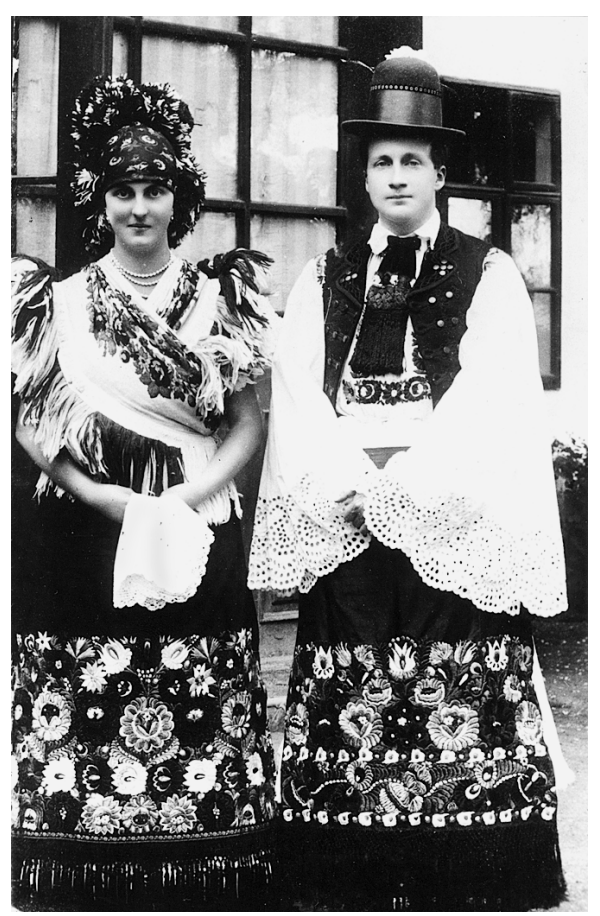

Abb. 10. Erzherzog Josef Franz und seine Gemahlin, Anna in Mezőkövesd im Jahre 1926, in Volkstracht gekleidet

rakteristik der Matyós kennenlernen konnten. In den Vitrinen des Gasthauses wurde den Gästen auch eine Handarbeitkollektion vorgezeigt.

6. Natürlich gab es Möglichkeit auch, Handarbeit, Volkstrachtpuppen, Ansichtskarten und andere Souveniere im Zeichen der Volkskunst zu kaufen. Es bat den Besuchern die Illusion originale Volkskunst zu haben. Mit der Herstellung und dem Verkauf der Handarbeiten beschäftigten sich in Mezőkövesd sowohl die Matyós, als auch Kaufleute, sogar zahlreiche Vertreter der lokalen Intelligenz seit dem Jahrhundertanfang. So konnte der Besucher wählen, ob er aus dem Geschäft der „HerzStickers" auf dem Hauptplatz, oder aus dem Angebot des Matyóhauses oder von Tante Bori Kis Jankó Handarbeit als Andenken kaufen möchte (Abb. 12).

„Das Matyó-Volk ist genauso an den Fremden angewöhnt, wie an scharfem Paprika“ - schrieb die Zeitung „Felsőmagyarország“ (Oberungarn) 1936. ${ }^{24}$ In demselben Jahr wurden 7000 Gäste ins Gastbuch des Matyóhauses eingetragen (Abb. 11).

Um die Zusammensetzung des Fremdenverkehrs in Mezókövesd zu veranschaulichen und illustrieren, sollen einige Beispiele aus der Reihe der vornehmeren Gäste

${ }^{24}$ Felsőmagyarország (Oberungarn), 6. Nov. 1936. 


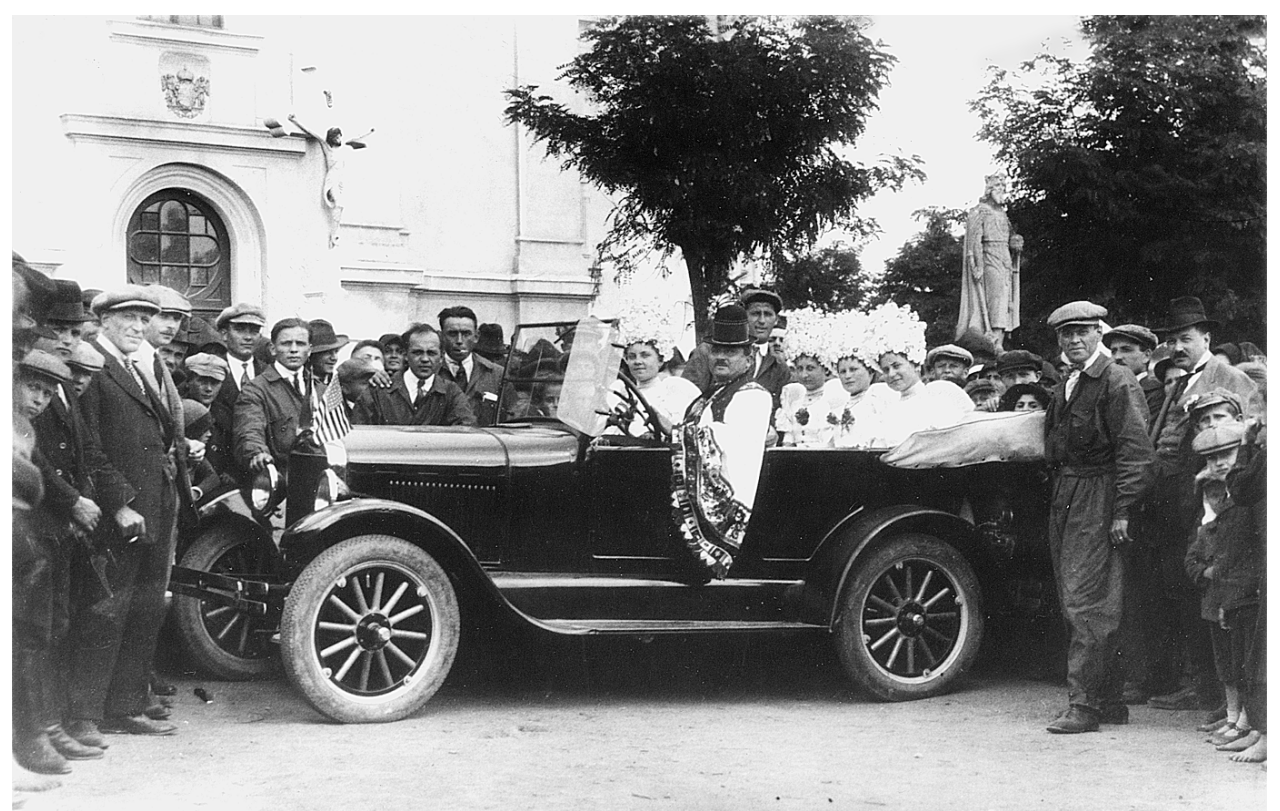

$A b b$. 11. Fremdenverkehr in Mezőkövesd. Englische Gäste und Marienmädchen im „autocar“

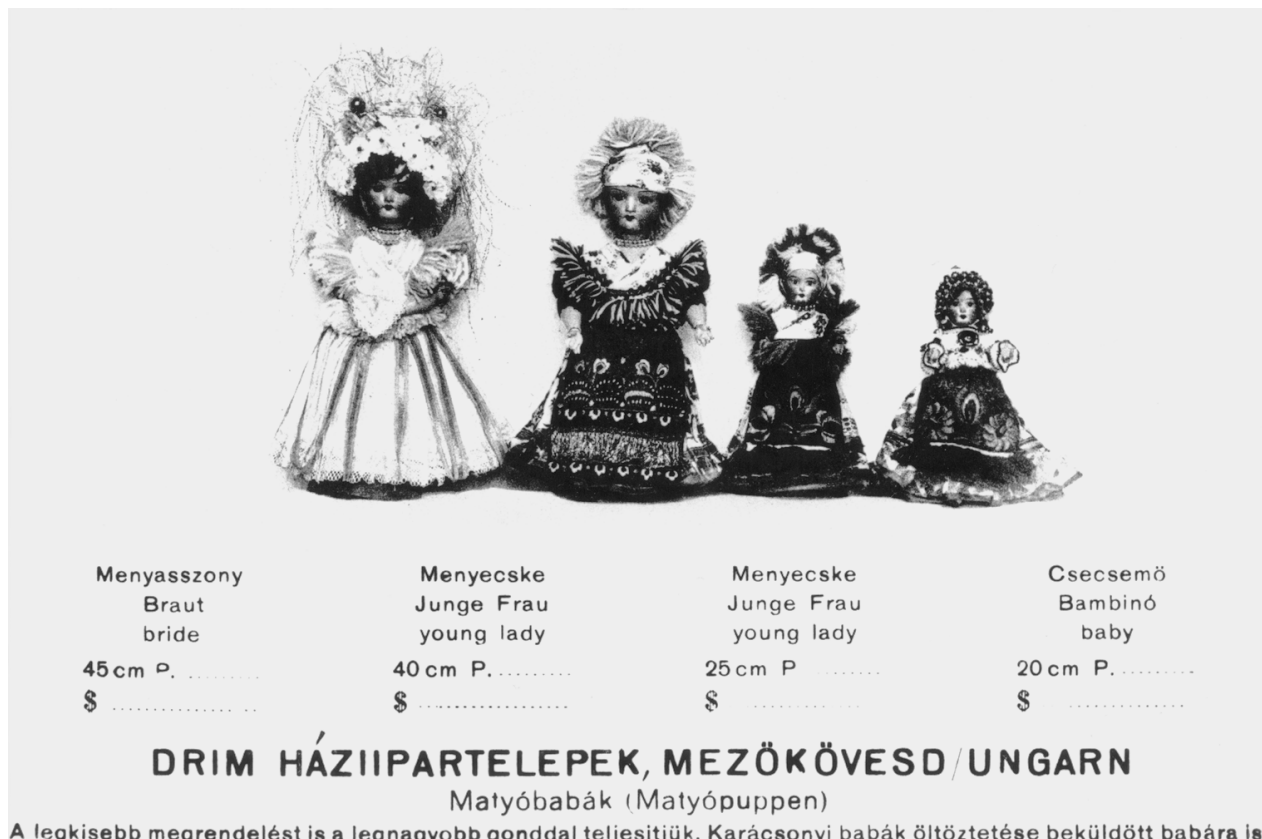

Abb. 12. Bestellungskarte mit mehrsprachiger Aufschrift, für Volkstrachtpuppen in verschiedener Grösse 
hier stehen. Die Zeitungen aus den 1930-er Jahren berichten unter anderen folgende: 25

Der Maharadscha von Indore, der reichste Fürst von Indien und sein Gefolge hat vor allem den wunderbaren Tanz in Mezőkövesd bewundert. Der Herzog von Windsor und seine Frau nahmen an einem Brautfest teil, der japanische Gesandte tanzte Tschardas im Matyóhaus. 250 deutsche Gäste kamen mit einem Sonderzug des Fremdenverkehrsbüros an, von dem Bahnhof fuhren sie mit einer Reitertruppe auf den Hauptplatz, und im Matyóhaus konnten sie die Volkstracht und die Volksbräuche gründlich kennenlernen. Die Teilnehmer der internationalen Frauenwoche, die Gäste des Budapester Finnisch-ugrischen Kongresses, die polnische Delegation des Báthory-Sobieski Festes haben unvergässliche Stunden mit Volkserlebnis in Mezőkövesd verbracht.

Von den vielen berühmten Personen, die Mezókövesd besuchten, war vielleicht der Besuch des Königspaares von Siam am denkwürdigsten. ${ }^{26}$ Die Figur des exotischen Herrscherpaares wurde in der Erinnerung der Einwohner foklorisiert. Der Besuch des Herrschers von Siam (heute Thailand) fand am 13-sten August 1934 statt, die begeisterten Vorbereitungen sowie die bedeutende Pressekampagne setzten die ganze Bevölkerung in Bewegung. Ein Prachttor wurde am Rande der Siedlung aufgebaut, auf dem Hauptplatz wurden zwei Throne unter einem mit Matyó Stickerei verzierten riesigen Laubzelt gestellt, wo das Königspaar die Brautfestvorstellung zu ihren Ehren besichtigen konnte. Inzwischen begann es nämlich stark $\mathrm{zu}$ regnen, und das veränderte das sorgfältig vorbereitete Programm, und das Brautfest wurde endlich in dem Katholischen Kreis nur vor einigen Gästen vorgeführt. Trotzdem war der exotische königliche Besuch für lange Zeit ein gutes Gesprächsthema des Volkes.

Die National Geographic veröffentlichte 1938 einen langen Artikel über das Matyótum mit dem Titel „Ein Sonntag in Mezőkövesd“ mit 15 Fotos illustriert. ${ }^{27}$ Der Autor, Margera Rae, versucht eine objektive und ausführliche Beschreibung über ihre Erlebnisse zu geben: „An Mezőkövesd denke ich, als eine typisch ungarische Siedlung, wo der Reisende das echte Bauernleben des ungarischen Landes erfahren kann" - schreibt sie. Trotzdem bewunderte sie vielerlei Sachen als etwas Romantisches, für einen Amerikaner natürlich unbekannte und unverständliche Erscheinungen. Der vor der Kirche trommelnde Gemeindediener, „der mündlich berichtende Journalist“, ist so eine Attraktion für einen Gast von Amerika. Der Kirchenbesuch am Sonntag und der Spaziergang der Mädchen Arm in Arm als die lokale Parade der Matyós erinnert die Korrespondentin an einen wunderschönen Blumengarten. Der Bauernofen, als „eigentümliches ungarisches Heizungssystem“ wird lange beschrieben. Objekt der Bewunderung ist das hochgemachte Bett, das den Autor trotz der gründlichen Beschreibung mit nichts auf der Erde vergleichen

${ }^{25}$ Berichtensammlung von Kálmán TóTH. In: Ethnographische Dokumentation des Museums Ottó Herman. Inv. Nr: 4680.

${ }^{26}$ Magyar Jövő (Ungarische Zukunft), 14. Aug. 1934.

${ }^{27}$ Ethnographische Dokumentation des Museums Matyó in Mezőkövesd Inv. Nr: 251. 
kann. Die spektakulären Stücke der Volkstracht erwecken natürlich das Interesse eines Fremden, das Burschenhemd wird mit dem Kimono verglichen, der Jungferkranz der Maria-Mädchen erinnert sie an den Kopfschmuck der Herzoginnen in Indien. Besonders phantasievoll sind die Beschreibungen der Abbildungen, wo sie versucht, das Leben der so weit lebenden exotischen Volksgruppe vorstellbar zu machen.

Mezókövesd und die Volkskunst der Matyós wurden also in den 1930-er Jahren zum ständigen und populären Angebot des ungarischen Fremdenverkehrs. Staatsmänner, Mitglieder internationaler Konferenze, Protokollgäste, Touristengruppen und Intellektuellen besuchten in grosser Menge Mezőkövesd, da sie sich ein ungarisches Volkserlebnis wünschten. Mit dem grossen Erfolg der Perlenstrauss-Bewegung gingen auch die Matyós regelmässig von ihrer Heimatsstadt aus, und brachten selbst die gewählten Werte der ungarischen Volkskultur den Stadtbürgern zur Stelle.

Der folgende Artikel aus dem Fachblatt „Fremdenverkehr“ 1934 ist eben in seiner Absurdität charakteristisch: „Es gibt Ausländer, die - wenn es um Ungarn geht - genau wissen, dass wir eine wunderschöne Hauptstadt haben, die in der Mitte von Matyóland liegt. “28 Die Begriffe wie Matyónien, Matyóland wurden vom Beginn des Jahrhunderts an mit dem ganzen Ungarntum identisch. Wie es in einem Bericht aus dem Jahre 1934 steht:"Mezőkövesd ist heute nicht nur in den Augen von Ungarn, sondern auch in den Augen ganzen Europas der wichtigste Repräsentant des ungarischen Dorfes, das eine echte Fundgrube der Einzelheiten für den Fremden und eine Menge Probleme für die Einwohner bedeutet.“

\section{LITERATUR}

K. CSILLÉRY, Klára

1983: Folklorizmus, historizmus és továbbélés egy Kalocsa környéki falu népmúvészetében. (Folklorismus, Historismus und Weiterleben in der Volkskunst einer Siedlung in der Nähe von Kalocsa.) Ethnographia XCIV. 353-382.

DALA, József-ERDÉLYI Tibor

o.J.: Matyóföld. A híres Mezókövesd. (Matyóland. Das berühmte Mezőkövesd.) Budapest (1941).

FEJős, Zoltán

1984: "Budapest Matyóország fővárosa“. (Budapest, die Hauptstadt von Matyóland.) In: Cul/tours (Hrsg. Zoltán FEJôs). Budapest, 40-43.

1991: Népmúvészeti divat a múlt század végén és a századeloón (Die Volkskunstmode am Ende des vorigen Jahrhunderts und am Jahrhundertsanfang.) In: Népi kultúra és nemzettudat (Hrsg. Tamás HOFER), Budapest, 143-158.

FLÓRIÁN, Mária

1990: A „sárközi szőttes“ története. (Die Geschichte der Weberkunst von Sárköz.) Ethnographia CI. $194-256$.

FÜGEDI, Márta

1997: Mítosz és valóság: a matyó népmúvészet. (Mythos und Wahrheit: die Volkskunst der Matyós.) Officina musei 6. Miskolc, 207.

1999: Aktionen, Bewegungen für Bewahrung und Konservierung der ungarischen Volkstracht in den Zwischenkriegsjahren. 6th Finnish-Hungarian Symposium in Ethnology 1997. (In Veröffentlichung).

${ }^{28}$ FEJŐs 1984: 42. 


\section{HOFER, Tamás}

1989: Paraszti hagyományokból nemzeti szimbólumok - adalékok a magyar nemzeti múveltség történetéhez az utolsó száz évben. (Von Volkstraditionen bis zur Nationalsymbolen-Angaben zu der Geschichte der Nationalkultur in den letzten hundert Jahren.) Janus VI. Pécs, 59-84.

1995: Bevezető: témák és megközelítések. (Einleitung: Themen und Annäherungen.) Néprajzi Értesítő LXXVII. 7-22.

F. KÁROLYI, Alexander

o.J.: Hungarian Pageant. Budapest, 113.

KozMA, Béla

1939: Matyóország. (Matyóland) In: Borsod vármegye. Vármegye szociográfiák V. (Hrsg. Antal CSIKVÁRY.) Budapest, 108-119.

PÁlfi, Csaba

1970: A Gyöngyösbokréta története. (Die Geschichte der Perlenstrauss-Bewegung.) In: Tánctudományi Tanulmányok (Hrsg. Gedeon DIENES-László MAÁCZ). Budapest, 115-161.

ROMSICS, Imre

1996: Gyöngyösbokréta és népviselet együttélése a kalocsai szállásokon. (Perlenstrauss und Volkstracht in den Siedlungen von Kalocsa.) In: Viselet és történelem - viselet és jel. (Hrsg. Zsuzsa KORKES), Aszód, 78-94.

SZABÓ, Zoltán

1938: Cifra nyomorúság. (Glänzendes Elend.) Budapest.

SZILÁGYI, Miklós

1980: Adatok a sárközi népmúvészet felfedezéséhez. (Angaben zu der Entdeckung der Volkskunst von Sárköz.) Dunatáj, No. 4.15-21.

TORMAY, Géza

1937: Népmúvészet és idegenforgalom. (Volkskunst und Fremdenverkehr.) In: A magyar idegenforgalom évkönyve, Budapest, 86-89.

ÚJVÁRI, Ferenc

1981: A Gyöngyösbokréta Boldogi Csoportjának története. (Die Geschichte der Perlenstrauss-Gruppe von Boldog.) In: A folklór helye a mai népéletben. A népmúvészeti együttesek mint múvelődési közösségek. (Hrsg. Péter MORVAY) Kecskemét, 1981. 21-37. 